\title{
UNA praxis agrícola, dialógica y ecosistémica
}

\section{UNA's ecosystemic and dialogic praxis}

Silvia Elena Berrocal-Montero

Universidad Nacional

Heredia, Costa Rica

silvia.berrocal.montero@una.ac.cr

iD https://orcid.org/0000-0002-8454-9783

José Alonso Calvo-Araya

Universidad Nacional

Escuela de Ciencias Agrarias

Heredia, Costa Rica

josealonsocalvoaraya@gmail.com

iD https://orcid.org/0000-0001-7294-6426

Allan González-Herrera

Universidad Nacional

Heredia, Costa Rica

allsolo7@hotmail.com

Martha Orozco-Aceves

Universidad Nacional

Heredia, Costa Rica

martha.orozco.aceves@una.cr

iD https://orcid.org/0000-0001-6223-912X

Marianela Rojas-Garbanzo

Universidad Nacional

Heredia, Costa Rica

iD https://orcid.org/0000-0001-7182-6975

Seiling Vargas-Villalobos

Universidad Nacional

Heredia, Costa Rica

seiling.vargas.villalobos@una.cr

Marisol Vidal-Castillo

Universidad Nacional

Heredia, Costa Rica

marisol.vidal.castillo@una.cr 
Resumen. En Costa Rica, la producción de hortalizas se ha intensificado en las últimas décadas por el uso de paquetes tecnológicos (fertilizantes y plaguicidas sintéticos) en monocultivos. Este modelo ha sostenido la producción en Zarcero, cantón que provee productos vegetales al Valle Central. Sin embargo, estudios realizados por el Instituto Regional de Estudios en Sustancias Tóxicas (IRET) han revelado resultados preocupantes sobre el uso de plaguicidas y sus efectos negativos en el ambiente y en la salud de las personas. Como respuesta a esta problemática se inició en el 2018 un proyecto con la participación del IRET, de la Escuela de Ciencias Agrarias y de la División de Educación Rural, cuyo objetivo es "promover un modelo alternativo en la producción agrícola mediante trabajo colaborativo, diálogo de saberes y la construcción social del conocimiento para disminuir el uso de agroquímicos en Zarcero, Alajuela".

En el proyecto se han desarrollado actividades con 1) equipo académico nacional e internacional, 2) personas agricultoras y sus familias, 3) escolares. El primer paso del proyecto ha consistido en la conformación de un equipo donde sus integrantes desarrollen: a) un marco teórico-político de referencia común, b) una mirada interdisciplinaria para analizar-trabajar-transformar la realidad y c) un tejido afectivo para favorecer entendimientos y desde ahí mancomunar metas. Para lograrlo, se han realizado: a) círculos de estudio, b) trabajo de campo, c) intercambio de saberes en el ámbito nacional e internacional, d) producción educativa agrícola modular y e) la articulación con la docencia, la investigación y la producción. Además, se ha posicionado la agroecología en las agendas políticas institucional, local, regional y nacional y en algunos territorios internacionales del continente.

Desde el trabajo académico tejido en el proyecto se ha aprendido a ser y a hacer "universidad necesaria", ya que las universidades requieren legitimarse en el país, por lo que se ha propuesto, para recuperar la confianza de las personas agricultoras y sus familias, gestar espacios de acercamiento para contextualizar conjuntamente y reflexionar sobre la problemática agrícola, revitalizar saberes locales y obtener insumos para la escritura de documentos educativos con enfoque sociocrítico desde la educación popular.

Palabras clave: educación, enfoque participativo, enfoque sociocrítico, plaguicidas, prácticas agrícolas.

Abstract: Costa Rica has intensified vegetable production by implementing monocultures managed with technological packages (synthetic fertilizers and pesticides). This model has sustained production in Zarcero, a municipality that supplies fresh vegetables to Costa Rica's capital and surroundings. However, investigations developed by the Regional Institute for Studies on Toxic Substances (IRET) have revealed concerning information on pesticide use regime in the area and 
URL: http://www.revistas.una.ac.cr/index.php/dialogo/index

CORREO ELECTRÓNICO: universidadendialogo@una.cr

DOI: http://doi.org/10.15359/udre.11-1.9

associated adverse effects on the environment and health of inhabitants. In response to this problem, IRET, the School of Agricultural Sciences, and the Division of Rural Education launched a project in 2018. The project's primary aim was "to promote an alternative model for agricultural production through collaborative work, social sharing and construction of knowledge, in order to reduce the use of agrochemicals in Zarcero, Alajuela." The project included activities developed by academic groups, farmers and their families, and school children. The academic groups worked to carry out the following activities: a) a theoretical-political framework of common reference; b) an interdisciplinary perspective to design work strategies based on analysis to transform actual situations; and c) good collaborative relationships, and from there to pool goals. Achieving these activities needed executing other ones: a) study circles; b) fieldwork; c) knowledge exchange processes at the national and international level; d) the production of educational written materials for farmers; and e) articulation of the previous activities with teaching, research, and production activities. In addition, agroecology has been positioned in the institutional, local, regional, and national political agendas and in some international territories. From the academic work produced in the project, we have learned to be and do "Necessary University" since public universities need to legitimize themselves in the country. Therefore, we proposed to regain the trust of farmers and their families by creating spaces for rapprochement to contextualize and reflect on agricultural problems jointly, revitalize local knowledge, obtain inputs for the writing of educational documents with a socio-critical approach from popular education. As a result, it has been possible to "sow" the idea of change in some farmers; spaces for exchange between local farmers and farmers from other municipalities have been created. Small children are a driving force for change in homes; so, we have actively worked with them guided by critical pedagogy and carrying out collaborative school-family-community work. Moreover, it has been possible to politicize the national events, re-signifying the farmer's knowledge and diversifying the learning scenarios. In conclusion, we consider that academic work linked to real situations enables improvement processes in the productive agricultural life of the communities and enriches teaching, research, extension, and writing in the public university. All these activities stimulate the social sharing of knowledge that links local knowledge to the academic one generated in universities, promotes unlearning of the traditional manner of "building university," and contributes to reflection anchored to the biocentric approach. The present work provides ideas for the construction of a university extension policy. It contributes to developing a work process to understand better the link that university life should have with the daily needs of communities and the processes of improving farming-socio-community life.

Keywords: agricultural practices, education, participatory approach, pesticide, sociocritical approach. 


\section{Introducción}

En Costa Rica (y el mundo) la producción de hortalizas se ha intensificado en las últimas décadas, debido al uso generalizado de paquetes tecnológicos que promueven el establecimiento de monocultivos, el uso de fertilizantes para proveer los requerimientos nutricionales a las plantas y plaguicidas para manejar las poblaciones de organismos no deseados.

Este modelo ha logrado consolidarse en Pacayas (Cartago) y Zarcero (Alajuela), ambos cantones son proveedores de productos vegetales frescos para personas consumidoras del Valle Central y demás regiones del país. Sin embargo, las consecuencias del modelo agrícola vigente han sido cuestionadas desde hace varios años, debido a los efectos negativos crecientes en suelo, agua y biodiversidad, daños en la salud de personas aplicadoras y cultivadoras, sus familias, la comunidad y en general en todo el entorno ambiental y cultural.

Estudios realizados por el Instituto Regional de Estudios en Sustancias Tóxicas (IRET) evidenciaron situaciones preocupantes sobre el uso de fertilizantes y plaguicidas en Pacayas en el año 2009 y en Zarcero en el 2016. En este último cantón se determinó que los cuatro plaguicidas de mayor uso son plaguicidas altamente peligrosos (PAP), los cuales se caracterizan por poseer uno o más de los siguientes atributos: alta toxicidad aguda, alta toxicidad crónica, alta toxicidad ambiental (tóxico para peces, abejas u otro organismo), y estar incluido en convenios ambientales internacionales (PAN, 2016).

Estos plaguicidas se usan en dosis altas, incluso por encima de la recomendación del fabricante (por ejemplo, plaguicidas a base de clorotalonil se utilizan en una dosis promedio de $5,9 \mathrm{~kg}$ i.a./ha/ciclo, plaguicidas a base de mancozeb se utilizan en dosis promedio de 5,2 kg i.a./ha/ciclo) y son utilizados por una gran cantidad de personas agricultoras (por ejemplo, clorotalonil es utilizado por $78,8 \%$ de las personas agricultoras encuestadas, mientras que cipermetrina es utilizado por $61 \%$ de las personas agricultoras encuestadas). Algunos de los PAP fueron detectados en concentraciones variables en suelo (por ejemplo, clorotalonil, clorpirifos y flutolanil), donde pueden ser muy persistentes (en una finca se detectó DDT en suelo), en cuerpos de agua como nacientes, ríos, incluso tanques de almacenamiento de agua potable (Asada) (donde se detectaron concentraciones mínimas de clorotalonil y protiofos), y en hortalizas en concentraciones mínimas (por ejemplo, clorotalonil, clorpirifos y cipermetrina; en cumplimiento con la norma costarricense) (SFE, 2016); sin embargo, esto no garantiza la seguridad de las personas consumidoras. 
En Zarcero, no solo la cantidad de plaguicidas utilizados en la agricultura está siendo inadecuada, sino también las prácticas de manejo; por ejemplo, únicamente $30 \%$ de las personas agricultoras realizan algún tipo de monitoreo de organismos no deseados para realizar aplicaciones de plaguicidas, y más bien realizan la aplicación de estos de forma preventiva, aunque el cultivo no presente signos o síntomas de enfermedad.

Una práctica crítica para usar la cantidad adecuada de plaguicidas es la calibración de la aplicación, y únicamente un $25 \%$ de las personas agricultoras indica realizarla, como consecuencia, es posible que los plaguicidas se estén aplicando en exceso, liberando más cantidad de estas sustancias al ambiente de la que se requiere. En conclusión, la comunidad entera de Zarcero está siendo expuesta a sustancias peligrosas de manera consistente, lo cual pone en grave riesgo la vida ecosistémica del lugar. Una situación similar persiste en Pacayas.

En consonancia con el marco normativo institucional de la Universidad Nacional, en cuyo Estatuto Orgánico se indican los principios de a) Humanismo: "La UNA promueve la justicia, el bien común, el respeto irrestricto a la dignidad humana a los derechos de las personas" y de la naturaleza y b) Responsabilidad ambiental: "Mediante las diferentes formas de su quehacer sustantivo, la universidad promueve la protección y defensa de los diversos ecosistemas, a fin de asegurar su conservación para las futuras generaciones" (Estatuto Orgánico de la Universidad Nacional, 2015, p. 19). En el 2018 dio inicio un proyecto para responder a la problemática detectada en Zarcero.

En dicho proyecto han participado académicos y académicas de tres unidades: IRET, Escuela de Ciencias Agrarias (ECA) y División de Educación Rural (DER), así como estudiantes de las áreas agrícola, biológica y ambiental, quienes han aportado a la consecución del objetivo: "Promover un modelo alternativo en la producción agrícola mediante trabajo colaborativo, diálogo de saberes y la construcción social del conocimiento para disminuir el uso de agroquímicos en Zarcero, Alajuela".

La problemática del uso inadecuado de agroquímicos en la agricultura no se reduce al quehacer de las personas agricultoras, ya que está altamente influenciada por tendencias y demandas del mercado nacional e internacional, e intereses ligados al capital por parte de corporaciones transnacionales, por lo tanto, se trata de una situación compleja y difícil de contrarrestar. Así lo expresan instituciones gubernamentales, universidades públicas, organizaciones no gubernamentales y organizaciones de la sociedad civil, que han realizado importantes esfuerzos para educar y concientizar sobre el uso 
y manejo adecuados de agroquímicos, pero es recurrente escuchar que son escasos los resultados positivos.

Debido a lo anterior, llegamos a la conclusión de que, para cumplir con el objetivo de nuestro proyecto, era crítico abordar la problemática desde un enfoque interdisciplinario y no exclusivo de las ciencias agrícolas, teniendo como eje transversal el pensamiento crítico. Por lo tanto, además de especialistas en Ciencias Agrícolas, fue necesario incorporar participantes de Educación Rural, Salud Ocupacional y Antropología.

Actualmente, nuestro equipo de trabajo está conformado por nueve académicas y académicos de la UNA y diez estudiantes, que han colaborado por tiempos finitos o de manera más constante. Las y los estudiantes han participado en el proyecto realizando prácticas profesionales supervisadas, como asistentes, y actualmente cuatro están realizando trabajos finales de graduación.

Es importante mencionar que el proyecto, además de tener el componente de extensión (con personas agricultoras, comunidad educativa), también contempla actividades de investigación (en tecnologías limpias: biocamas, biorremediacion, biojardineras), docencia (jornadas de trabajo con estudiantes de grado y posgrado) y producción (material didáctico para escolares y módulo educativo dirigido a personas agricultoras).

Además, se logró incorporar la temática de agroecología en el rediseño de la oferta curricular donde se forman educadores y educadoras que laboran en contextos rurales de diversas regiones del país.

\section{Metodología}

En el proyecto, la metodología es participativa y se fundamenta en un enfoque sociocrítico (Santaella, 2014) que parte de problematizar la realidad y se esfuerza por forjar referentes alternativos para reflexionar-transformar la práctica agrícola comunitaria. De manera general, la metodología se ha construido en conjunto durante dos años y seis meses y se ha desarrollado en tres niveles; sin embargo, estos no son excluyentes, por el contrario, se traslapan y realimentan unos a otros. Los niveles de trabajo son: 1) equipo académico y estudiantes, 2) personas agricultoras, sus familias y comunidad, 3) comunidad educativa y Gobierno local. 
URL: http://www.revistas.una.ac.cr/index.php/dialogo/index

CORREO ELECTRÓNICO: universidadendialogo@una.cr

DOI: http://doi.org/10.15359/udre.11-1.9

El detalle de las metodologías se describirá a continuación.

\section{Trabajo con equipo académico y estudiantes}

El primer objetivo que planteamos en el proyecto fue "formar un grupo multidisciplinario de académicos preparados para el desarrollo de procesos educativos participativos, no formales dirigidos a productores agrícolas". Este surge como respuesta a la pregunta: ¿Tenemos los académicos y las académicas de la UNA las habilidades para conformar equipos interdisciplinarios y llevar a cabo proyectos de extensión en las comunidades orientados por el pensamiento crítico?

Con base en esto, las primeras actividades estuvieron encaminadas a desarrollar un marco teórico-político de referencia común, una mirada interdisciplinaria para analizar-trabajar la realidad y un tejido afectivo para favorecer entendimientos entre los y las integrantes del grupo. Para tratar de lograr lo anterior, se implementó la metodología del círculo de estudio.

Esta actividad se realiza de manera quincenal desde el inicio del proyecto en enero de 2018 hasta la fecha, y se planea continuar durante todo el 2021. En el 2018, durante los círculos de estudio, las personas del equipo académico (académicos, académicas y estudiantes) preparaban y exponían temas de interés, recomendaban lecturas o teledocumentos para lectura independiente, o se sugería la intervención de personas invitadas, las temáticas debían estar vinculadas al quehacer del proyecto. Independientemente de la modalidad, durante cada sesión se desarrollaba una discusión y un análisis de los temas.

Algunos ejemplos de temas tratados durante los círculos de estudio son: fundamento sociopolítico de la universidad necesaria, exposición de los principales resultados obtenidos en el proyecto Las buenas prácticas agrícolas en el uso y manejo de agroquímicos en la zona hortícola de Zarcero, Alajuela, realizado entre 2014 y 2016, experiencias educativas con productores, metodologías participativas, salud ocupacional, enfermedades de cultivos, ecología de suelos, control biológico, mirada crítica al sistema capitalistaextractivista, la organización y participación social en la vida local, entre otros.

Durante el 2019, los círculos de estudio se enfocaron en la generación de un pensamiento académico que favoreciera la construcción de materiales educativos que servirá como material de apoyo para el trabajo con personas 
agricultoras en fases posteriores del proyecto. Lo anterior, con la finalidad de cumplir el objetivo: "Diseñar un módulo de educación continua dirigido a personas productoras agrícolas sobre uso y manejo de plaguicidas para su implementación en Zarcero, Alajuela". Hasta la fecha se han realizado sesenta círculos de estudio.

A lo largo de la vigencia del proyecto se ha implementado la metodología de trabajo de campo en Zarcero. Se realizan giras de forma quincenal, a las cuales asisten tanto académicos y académicas como estudiantes; sin embargo, desde marzo de 2020, a raíz de la pandemia, el trabajo de campo demandó ajustes en la programación para apoyar la comercialización de algunas cosechas de familias agricultoras con las que se ha trabajado articulando la investigación y la extensión; se tuvo que bajar la frecuencia de las giras por la imposibilidad de contar con el transporte necesario en el ámbito institucional, debido a directrices sanitarias, pero gracias a la disposición de personas en el equipo se han realizado giras para brindar acompañamientos en momentos de incertidumbre, crisis y temor.

También se han realizado giras a Los Santos, a Heredia y a nivel internacional a Bolivia en el 2018 y Colombia en el 2019. Los objetivos de las giras de campo han sido distintos en diferentes etapas del proyecto. En una primera etapa, en el 2018, la intención de las giras fue presentarnos como equipo de trabajo ante las personas agricultoras, informarles sobre los resultados del proyecto anterior (realizado entre el 2014 y el 2016) e invitarles a participar en el nuevo proyecto.

Durante esta primera etapa fue posible conocer que la UNA y en general las universidades están deslegitimadas en la comunidad de Zarcero, esto ha sido un reto para el equipo, ya que se ha tenido que trabajar recuperando la confianza de las personas agricultoras en los procesos de trabajo académico que se generan desde la educación superior. Para esto, realizamos visitas a las fincas; ahí pasamos un tiempo trabajando en las actividades agrícolas que estuvieran realizando las personas agricultoras al momento de la visita (fertilizando, cosechando).

Esta sencilla tarea logró abrir espacios de acercamiento para contextualizar conjuntamente y reflexionar sobre la problemática agrícola, revitalizar saberes locales, conocer problemáticas y satisfacciones de la actividad, preocupaciones, incertidumbres y decepciones derivadas del trabajo agrícola. Hasta el momento hemos realizado aproximadamente cincuenta giras a Zarcero. 
En el 2019, en una tercera etapa de trabajo de campo, nos hemos enfocado en recopilar material para la construcción del módulo educativo, el cual se está diseñando en coautoría con las personas agricultoras de Zarcero. En dicho módulo, se recopila y presenta información técnica, conocimiento local, opiniones, pensamientos y sentires de las personas visitadas durante las giras. El módulo educativo toca diez temas generales, algunos ejemplos son: conocimiento y protección del suelo, sistemas alternativos de producción, problemas y amenazas para la agricultura, enfoque integral de la seguridad y salud laboral en la agricultura, manejo fitosanitario de cultivos hortícolas, agricultura familiar y uso y manejo adecuado de plaguicidas.

Otra actividad importante realizada durante el 2018 y el 2019, ha sido la presentación de información del quehacer del proyecto en diversos foros y ante diferentes públicos de los ámbitos institucional, local, regional, nacional e internacional, como acción académica de ir logrando espacios en las agendas político-decisorias en diversos escenarios.

Además, estratégicamente en el proyecto se han incorporado estudiantes de diversas carreras de la UNA en las actividades. Las y los estudiantes que nos han acompañado cursan las carreras de Ingeniería en Gestión Ambiental, Ingeniería Agronómica y Biología. También, se han enriquecido las temáticas de los cursos desarrollados a nivel de grado y posgrado con literatura, teledocumentos y experiencias de campo aportadoras de un saludable debate en cuanto a las condiciones en que debe desarrollarse la producción agrícola rural para promover comunidades saludables.

\section{Trabajo con personas agricultoras, sus familias, comunidad $\mathbf{y}$ Gobierno local}

Además del trabajo realizado con las personas agricultoras durante las giras al campo, hemos organizado diversas actividades con enfoque persona agricultorafamilia-comunidad. Lo anterior bajo la premisa de que los y las integrantes de la familia (parejas, hijos e hijas), a pesar de que en muchos casos no participan directamente de las decisiones que se toman en las fincas, sí influyen de manera indirecta en el funcionamiento de estas. Por ejemplo, las parejas (sobre todo las mujeres), los hijos y las hijas son una fuerza promotora de cambios.

Ejemplos de estas actividades son: 1) Una gira con familias participantes del proyecto a la Finca Educativa Don Juan, donde se promueve la agricultura ecológica, la agricultura familiar y diversos procesos educativos de cuidado 
de la Madre Tierra. 2) Dos convivios intercantonales Zarcero-Los Santos y Buenos Aires de Puntarenas-Zarcero donde familias de ambos cantones visitaron la finca ecológica Tierra de Sueños en Zarcero para conocer más de los principios de la agroecología y discutir el tema del relevo generacional en la agricultura. Fue un espacio que politizó (en el buen sentido) el tema al demandar gobernanzas locales desde experiencias agrícolas alternativas.

En lo que resta de la vigencia del proyecto se plantea la realización de algunas actividades orientadas por el enfoque persona agricultora-familia-comunidad con el objetivo de co-diseñar (presentar), llevar a la práctica, realimentar y validar el material educativo que se está terminando de escribir.

Importante mencionar que una iniciativa del proyecto fue presentar una propuesta de cambio al Reglamento de contrataciones de la UNA, para incorporar la figura de la "persona con saberes locales, ancestrales o experienciales". Lo anterior en respuesta al hecho de que el trabajo académico desarrollado desde un enfoque participativo en el proyecto ha permitido comprender, reconocer y valorar epistemologías alternativas ancladas a la vida cotidiana de las familias y comunidades, por lo que se propone incorporar a personas con saberes locales a compartir y deconstruir espacios de vida universitaria.

Desde el proyecto reconocemos que la sociedad nacional y continental demanda una universidad pública inteligente, pensante, reflexiva, facultada para construir y deconstruir su cultura desde un diálogo aprendiente afincado en las necesidades de las comunidades; lo anterior implica robustecer la universidad necesaria, para demostrar al país que la UNA es capaz de forjar un trabajo donde se encuentra el conocimiento generado en la investigación universitaria con los saberes atesorados en la práctica cotidiana de la vida de las familias y comunidades productoras agrícolas.

Es relevante considerar que en el espacio universitario contemporáneo se devela una crisis epistémica-social, política, económica-ambiental, donde se demanda estudiar lo académico a fin de construir una mirada crítica intrauniversitaria, que posibilite replantear con responsabilidad y compromiso los pilares en que tradicionalmente se ha fundamentado la manera de "hacer universidad" (Carta a la comunidad universitaria, 2018).

Desde el trabajo académico deconstruido podemos expresar que es necesario incorporar personas con saberes locales a la vida académica de la UNA, para enriquecer y revitalizar el trabajo que se demanda en la docencia, investigación, extensión y producción desde la universidad necesaria. Como cuerpos académicos tenemos el deber de construir la UNA como una organización sensible, versátil, 
URL: http://www.revistas.una.ac.cr/index.php/dialogo/index

CORREO ELECTRÓNICO: universidadendialogo@una.cr

DOI: http://doi.org/10.15359/udre.11-1.9

inteligente, coherente y fielmente comprometida con la mejora de la vida en las comunidades, por lo que necesitamos un centro de estudio superior capaz de diseñar procesos formadores alternativos, donde se forjen diálogos animadores de desaprendiencias de esa episteme colonizadora, que invisibiliza el saber que construyen los pueblos y las personas lejanas a la academia.

Las áreas sustantivas asumidas desde la universidad necesaria tienen la misión de fraguar un imaginario social incluyente, justo, solidario, donde sea posible tejer un sentido de vida alternativo, vinculado al logro de comunidades luchadoras, resilientes, emancipadas, cultivadoras y deseosas de combatir las dependencias agroalimentarias.

La sociedad nacional e internacional demanda instituciones de educación superior dispuestas a compartir espacios de diálogo-aprendiente, desde visiones decolonizadas y desde acciones comprometidas.

Dietz, citado por Casado et al. (2015), expresó que

La universidad del futuro no puede ser un contenedor de conocimiento sino una instancia para el diálogo entre distintos saberes que frente al pretendido monopolio del conocimiento por parte de una institución que se postula clave para la transformación de cualquier instancia excepto de sí misma. (p. 26)

Por lo que para lograr legitimación a nivel intrauniversitario es esencial concretar coherencia entre el decir y el hacer; en este caso, es necesario comprender que el conocimiento se crea y recrea en diálogo permanente con las personas que tejen la vida y son capaces de resolver sus problemas desde el conocimiento generado de manera natural en sus experiencias de vida cotidiana.

Para lograr lo anterior, es relevante crear espacios de reflexión que permitan el encuentro intersubjetivo de personas dentro y fuera de la academia, a fin de generar alternativas facilitadoras de cambios y transformaciones para el logro de un mejor país. Esto contribuirá a formar las personas que van a tener la responsabilidad de aportar a la reconstrucción de una sociedad donde se ven reflejados serios problemas en diversos ámbitos: social, económico, político, ambiental y, de manera relevante, en lo ético.

La sociedad contemporánea demanda nuevas rutas en la formación universitaria, no se puede pretender cambios sustantivos en la sociedad, si no se transforman las instituciones y, de manera particular, la manera en que se desarrollan los procesos de vida universitaria. 
En términos prácticos, existe en elámbito local personas con saberes significativos para enriquecer la vida académica y los procesos de vida universitaria, así como la vida comunitaria, por lo que una gestión académica alternativa permite legitimar saberes que construyen las personas de la comunidad a partir de las experiencias de vida cotidiana y la articulación de estos aprendizajes con referentes teóricos. Esto es también fundamental para disminuir la brecha entre la universidad y la sociedad, brindando evidencias de un acercamiento respetuoso, dialógico y comprometido con la vida local, nacional e internacional.

\section{Trabajo con la comunidad educativa de la Escuela La Brisa}

La escuela rural históricamente ha tenido una fuerza educadora mediante la articulación de su quehacer con las familias y la comunidad; es por este motivo que desde el proyecto se consideró un trabajo político-pedagógico para revitalizar la idea de cuidar, respetar y proteger la Madre Tierra y, de manera particular, transformar las prácticas de cultivo agrícola en el cantón de Zarcero como referente para otras regiones del país y del continente.

El trabajo académico demandó el diseño de círculos de estudio con el equipo docente de la escuela para forjar una mirada sociocrítica sobre el papel de la escuela ante la problemática de la vida local, de manera particular sobre las prácticas de cultivo en la comunidad.

Desde el concierto de voces docentes e infantiles se diseñaron diversos espacios educativos que permitieron reflexionar sobre la idea de escuela caminante; es así como fue posible viajar a otros territorios en el ámbito nacional e internacional para compartir experiencias e intercambiar ideas que reanimen la vida de la escuela y generen cambios positivos en la vida de la comunidad.

Desde el marco animador que brinda la pedagogía crítica fue posible transformar el enfoque de las efemérides escolares, para reposicionar en la agenda escolar el debate político-educativo tendiente a favorecer un sentido de vida alternativo, donde sea posible comprender y valorar la relacionalidad, interdependencia, reciprocidad y complementariedad que fundamentan la vida en la Madre Tierra.

Gracias al trabajo educativo en la Escuela se logra vivir una experiencia pedagógica que desdibujó la frontera escolar; además, posibilitó escribir-vivir una ruta metodológica para animar procesos formativos en la escuela rural y permitió producir un documento que registra el trabajo pedagógico logrado con la comunidad educativa que se animó a diseñar alternativas pedagógicas vinculadas a la vida productiva saludable, responsable y sustentable. 
Las voces infantiles generaron un valioso espacio de reflexión y acción social vinculado al tema proteger la salud humana y del ambiente, dada la legitimidad que su pensamiento tiene ante la escuela, la familia, la comunidad y la universidad.

Este material educativo fue socializado en pueblos hermanos del continente gracias a la coordinación con egresados y egresadas de la Maestría en Educación Rural Centroamericana de la UNA y a la gira internacional realizada a Colombia en el 2019.

\section{Resultados vinculados a la construcción de la política de extensión}

Desde el trabajo de extensión que hemos realizado en el marco del proyecto, consideramos que los elementos críticos a tomar en cuenta para realizar extensión desde la universidad necesaria son los siguientes:

\section{Considerar los fines fundacionales de la UNA}

El trabajo de extensión de las universidades y en especial el trabajo realizado desde la UNA como universidad necesaria debe estar cimentado en los fines fundacionales institucionales, en otras palabras, debemos ser claros en responder a la pregunta: ¿Para qué existe la extensión desde la UNA? Para dar respuesta a lo anterior, se requiere conocer e incorporar en los proyectos de extensión los principios, valores y fines considerados en el Estatuto Orgánico.

Este aspecto pudiera parecer obvio, pero la realidad es que la mayoría de personas académicas lo desconoce. Los círculos de estudio y el trabajo de campo interdisciplinario realizados en nuestro proyecto nos han permitido comprender el qué, cómo, por qué y para qué se realiza la extensión, para lograr una sociedad robusta en justicia social. Es decir, el trabajo en el proyecto nos ha permitido comprender cómo debemos construir la vida académica para dar vida a la Universidad Necesaria. Algunos principios, valores y fines institucionales que incorpora nuestro proyecto son: la responsabilidad ambiental, el diálogo de saberes, la interdisciplinariedad y el pensamiento crítico.

\section{Incluir el enfoque sociocrítico}

El trabajo académico en la UNA debería ser construido desde el enfoque sociocrítico, el cual busca problematizar la realidad para generar conciencia social y proponer transformar la realidad para el bien de las poblaciones vulnerabilizadas, mediante una mirada rigurosa, cuestionadora, pero esperanzada, que prepondera 
los problemas que emergen de la vida cotidiana, lo que implica la participación de los actores sociales, y favorece la ruptura de la frontera disciplinar, tan arraigada en la academia. Lo que finalmente promueve una praxis dialógica bidireccional basada en la interdisciplinariedad y el diálogo de saberes.

El enfoque sociocrítico en la extensión requiere un vínculo estrecho con la realidad, lo cual enriquece y estimula la acción sustantiva de la universidad; sin embargo, constituye un gran reto debido a que es frecuente que los académicos y las académicas pongan resistencia a salir de su recinto y disciplina. Por último, el enfoque sociocrítico promueve la curiosidad epistémica, el espíritu estudioso, creativo, emancipado, que da sentido y fortaleza a la universidad necesaria.

En el proyecto hemos puesto en práctica el enfoque sociocrítico durante el trabajo de campo, con buenos resultados. El grupo interdisciplinario plantea ejercicios reflexivos basados en la teoría que animen diálogos con las personas agricultoras y en conjunto se contextualiza alguna problemática relacionada con las prácticas agrícolas inadecuadas realizadas en sus fincas. Con este ejercicio se ha logrado "sembrar" la idea del cambio en algunas personas agricultoras, se han creado espacios de intercambio locales e intercantonales. Además, se ha recopilado material e información para la construcción de material educativo en coautoría de las personas agricultoras de Zarcero.

\section{Articulación de áreas sustantivas}

La extensión universitaria desarrollada desde el enfoque sociocrítico brinda elementos para realimentar y articular la docencia, la investigación y la producción académica. Algunas actividades que ejemplifican la articulación de las áreas sustantivas de la UNA sobre el eje de la extensión son:

A) Incorporación de estudiantes en las actividades del proyecto en las modalidades de práctica profesional supervisada, trabajos de cursos, trabajos finales de graduación y estudiantes asistentes. Hasta el momento los y las estudiantes han desarrollado: a) dos prácticas profesionales supervisadas; i) Análisis de la gestión de los envases vacios de agroquímicos en Zarcero por Kimberly Mejía Alvarado, en colaboración con la Oficina de Gestión Ambiental de Zarcero, y ii) Evaluación del potencial de degradación de biocamas inoculadas con microorganismos descomponedores de clorotalonil en la finca experimental Santa Lucia, por Mary Paz Jiménez Domínguez; b) trabajo: Caracterización de cepas bacterianas con capacidad de 
degradación de plaguicidas, aisladas de suelos agrícolas de Zarcero, Alajuela, como investigación del curso Biotecnología aplicada por las estudiantes Joyce Estrada Gamboa, Johanna Ramírez Ruíz, Violeta Oliva Mercado y Stephanny Sánchez Vargas; c) dos trabajos finales de graduación, los cuales se encuentran en proceso: i) Estrategia para la disposición adecuada de residuos de plaguicidas generados por pequeños y medianos productores horticolas del cantón de Zarcero, 2019-2020, por Mary Paz Jiménez Domínguez y ii) Propuesta de diseño de un humedal construido para el tratamiento del agua residual de una finca empacadora de hortalizas en Zarcero, por Erika Montero Angulo; y dos trabajos finales de graduación que se comenzaron en el 2020: i) Biorremediación de suelos contaminados con plaguicidas como colaboración a un proyecto de Buenas Prácticas Agrícolas del Instituto Regional de Estudios en Sustancias Tóxicas (IRET), Zarcero, 2020, por César Jiménez Vargas, y ii) Evaluación de una estrategia de biorremediación de suelos en fincas hortícolas de Zarcero, Alajuela, por José Francisco Sibaja Arce. Por último, contamos con dos estudiantes asistentes: Joyce Estrada Gamboa y Jorge Berrocal Alfaro, quienes apoyan las actividades de investigación y extensión del proyecto.

B) Curso optativo ofrecido a estudiantes de la División de Educación Rural. El proyecto académico diseñó el curso optativo Ecología y Ética de los Cuidados, que aporta conocimiento para realimentarenriquecer-transformar el rediseño de la carrera de Licenciatura en I y II Ciclos con Énfasis en Educación Rural. Esta propuesta curricular para la formación de educadores y educadoras que laboran en zonas rurales e indígenas brinda un espacio de formación ciudadana con mirada crítica, para analizar tendencias macroeconómicas globales inspiradas en la producción de monocultivo y el impacto de este proceso en la desterritorialización de las comunidades rurales. En el plan de estudio, el curso se describe como "una experiencia educativa-ético-productiva para construir mirada y acción crítica en torno a las tendencias productivas contemporáneas, en aras de forjar una vida rural alternativa desde donde sea posible comprender y prorrogar los principios de relacionalidad, reciprocidad, interdependencia y complementariedad en los que se sustenta la vida en la Madre Tierra. Este curso es un espacio educativo que ayuda a forjar conciencia sociocomunitaria, que estimula la soberanía alimentaria, la producción de alimentos en armonía con el ambiente, 
la conservación y multiplicación de semillas criollas; y la autonomía en la producción de alimentos sanos para la familia, la comunidad, el cantón, la provincia y el país. Se espera con esta propuesta curricular generar espacios educativos formales que favorezcan la vivencia de procesos educativos con sentido y significado para la vida rural de las comunidades.

C) Módulo educativo, específicamente el material construido en conjunto con niños, niñas, educadores y educadoras de la Escuela La Brisa, emerge de la pedagogía crítica y del compromiso por generar mejoras en la vida de la comunidad a partir del pensamiento propositivo que emerge de la escuela como proyecto político comprometido con la vida comunitaria. El diálogo aprendiente anclado en la problematización de la vida productiva local, realimentado por el trabajo pedagógico propositivo de una escuela caminante permitió el diseño de una ruta metodológica que favoreció la producción de materiales educativos reveladores de las vivencias pedagógicas de la comunidad educativa donde se fortalece el vínculo escuela, familia y comunidad. Este material se llevará como obsequio en el 2021 a las personas agricultoras de la zona y al gobierno local, para expresar la valoración al trabajo agrícola y evidenciar el proceder acompañante de la escuela rural en la instauración de la producción agrícola sustentable.

\section{Trabajo interdisciplinario y diálogo de saberes}

Las problemáticas que emergen desde el enfoque sociocrítico durante las actividades de extensión deben ser abordadas desde la interdisciplinariedad y el diálogo de saberes, como se ha mencionado anteriormente. Gracias a estos elementos se posibilitó el encuentro de familias agricultoras, de población estudiantil universitaria, de autoridades comunitarias, de la escuela, la familia y la comunidad de Zarcero para reflexionar sobre problemas ambientales que se presentan en la comunidad, como el uso intensivo de plaguicidas, problemas de contaminación de agua, afectaciones a la salud, y otros, para proponer soluciones desde un enfoque ecosistémico.

Algunos ejemplos de actividades que se han realizado con este fin han sido: a) la visita del Concejo Municipal y de la comunidad de Buenos Aires de Puntarenas a la finca orgánica Tierra de Sueños en Zarcero, con el objetivo de conocer prácticas agrícolas ecológicas, b) la visita de personas agricultoras de Zarcero a la Finca Educativa Don Juan para conocer un proyecto ecológico 
integral en la Fortuna de San Carlos, c) el trabajo académico con personas invitadas internacionales que aportan al debate y a la definición de rutas metodológicas, d) además, se han abordado problemas de índole social y de importancia nacional, como es el relevo generacional en la agricultura, este tema fue discutido durante el Convivio Intercantonal Zarcero- Los Santos.

\section{Socialización en el ámbito nacional e internacional}

Es crítico que la información y el conocimiento que se generan en las universidades se dé a conocer tanto en los ámbitos institucional y comunal como a nivel nacional e internacional, mediante actividades de divulgación. Estas actividades son importantes porque son una vía mediante la cual se legitima el trabajo de las instituciones de educación superior.

Algunos ejemplos de actividades de divulgación que se han realizado en el marco del proyecto son: las presentaciones en el Consejo de Vicerrectores de la UNA y la Comisión Curricular del Centro de Investigación y Docencia en Educación (CIDE), charlas a estudiantes solicitadas por profesores y profesoras de diversos cursos de las Escuelas de Biología y de Ciencias Agrarias y una jornada de trabajo con estudiantes de la Maestría en Educación Rural Centroamericana (MERC), actividades en el marco de la visita del profesor pasante Omar Felipe Giraldo Palacio, del Colegio de la Frontera Sur (México), presentaciones en el Concejo Municipal y el Comité Sectorial Local de Zarcero, en la Reunión de Directores de escuelas y colegios de la regional de San Ramón, ante personas funcionarias del Servicio Fitosanitario del Estado, coordinación con televisora local, entre otros.

\section{Discusión y conclusiones}

El proceso académico universitario desarrollado en el proyecto donde participan tres unidades de dos facultades (Ciencias de Tierra y Mar y del Centro de Investigación y Docencia en Educación) ha enriquecido el enfoque participativo para problematizar la realidad global-nacional-local. Esto ha favorecido la construcción de una mirada crítica al sistema sociopolítico-económico y productivo mediante la constitución de equipos académicos interdisciplinarios que trascienden la mirada disciplinar para comprender, explicar, analizary aportar a la mejora de la vida en la realidad. Lo que permite un despertar de conciencia para descubrir la urgencia de forjar un sentido de vida alternativo en la familiacomunidad-sociedad, inspirado en los principios que rigen la vida en la Madre Tierra: relacionalidad, interdependencia, reciprocidad y complementariedad. 
Durante las actividades del proyecto tratamos de profundizar en el estudio de la interrelación de conocimientos experienciales y su vínculo con referentes teórico-conceptuales alternativos, lo que permitió dar sentido y significado político a la universidad necesaria, tanto en el ámbito nacional como internacional. Además, la ruta metodológica forjada en el proyecto devela un hallazgo relevante para decolonizar la vida académica en la UNA, por ejemplo, a través del sentir, el pensar y el hacer de la comunidad infantil, que legitima en conjunto con la comunidad un proceso de cambio en la manera de cultivar y establecer relaciones con la Madre Tierra.

A lo largo de dos años y medio nuestra concepción del trabajo en el proyecto ha ido evolucionando, y ha cambiado sustancialmente en comparación con el plan/idea inicial. De tal manera que, actualmente, nuestro trabajo aspira a resignificar el saber académico en la UNA a partir de las experiencias y vivencias compartidas entre nosotros y la comunidad de Zarcero, para dar paso a la construcción de una comuniversidad (Roldán, 2012). Este escenario permite el encuentro de saberes, donde se promueve una actitud dialógica que atienda las cualidades sensibles para entender las dialécticas superpuestas en la vida humana y su relación con el entorno.

Para lograr lo anterior reconocemos que es un gran reto para los académicos y las académicas que realizamos actividades de extensión, ya que se requiere un giro axiológico, desarrollar nuevas sensibilidades y alternativas de asumir y asumirse con el otro, un nuevo protagonismo de aprendientescomunidad-mediadores-actores educativos. Además de una participación con inclinaciones hacia la emancipación crítica, con conciencia, donde prevalezcan las relaciones horizontales, la otredad, la alteridad y el respeto a la diferencia, donde la persona se asuma como líder, generadora de espacios de intercambio de saberes que le permitan repensarse desde su interioridad y desde el quehacer formador en su conjunto.

En palabras de Maffesoli (2004), citada por Caldera et al. (2016):

La universidad debe ser el centro donde realmente dialoguen el arte, la cultura del pueblo, las expresiones de los jóvenes, los saberes cotidianos que reclaman su justo lugar, donde se reconozcan las metáforas de los voceros culturales vivientes en las experiencias académicas Experiencias que, como plantea Larrosa (2003), han sido minusvaloradas, entendidas como "conocimientos inferiores" que la ciencia moderna trató de objetivar, homogeneizar y controlar, convirtiéndola en experimento. (p. 10) 
La universidad necesaria es sensible y exige voluntad férrea de los actores socio-comunitarios, para trascender la dinámica de vida universitaria tradicional y conservadora, que desunen, aíslan y excluyen experiencias y trayectorias vitales de las comunidades costarricenses. Algunos otros autores relevantes que fundamentan la resemantización de la relación entre universidad y comunidad son: Foucault (1970), Martínez (2001), Téllez (2001), Maffesoli (2004), Larrosa (2003) y Dussel (2001).

Como conclusión se establece que el trabajo académico vinculado a la realidad posibilita procesos de mejora en la vida productiva agrícola de las comunidades y enriquece la docencia, investigación, extensión y producción universitarias, por cuanto estimula el diálogo de saberes, articula el saber local con el conocimiento generado en las universidades, impulsa la desaprendiencia en la manera tradicional de construir universidad y aporta a la reflexión anclada en lo biocéntrico.

Lo anterior da sentido y significado al fundamento de UNA praxis agrícola dialógica y ecosistémica. La contribución de este trabajo para la construcción de una política de extensión es fundamental, por cuanto aporta un proceso de trabajo que permite comprender el vínculo que debe tener la vida universitaria con las necesidades cotidianas de las comunidades, y los procesos de mejora en la vida socio comunitaria-productiva.

\section{Referencias}

Caldera, Y., Fernández, M. y Guevara, C. (2016). La academia sensible: experiencias y encuentro de saberes en la universidad venezolana. Telos, 18(1),4-16. https://www.redalyc.org/jatsRepo/993/99344833002/html/ index.html

Carta a la comunidad universitaria. (2018). Congreso Universitario: Transformando la Docencia en la UNA. Vicerrectoría de Docencia, Universidad Nacional. Heredia, Costa Rica. 31 p.

Casado, A. E., Ruiz, L. y López, C. C. (2015). Ecologías de saberes dentro de la universidad: propuestas de intervención para liberar dispositivos docentes. Profesorado, 19(2), 25-40.

Dussel, E. (2001). Hacia una filosofia política crítica. España: Editorial Desclée. 
Estatuto Orgánico de la Universidad Nacional. (2015). Heredia, Costa Rica. $114 \mathrm{p}$.

Foucault, M. (1970). Saber y verdad. España: Las Ediciones de la Piqueta.

Larrosa, J. (2003). La experiencia y sus lenguajes. Seminario Internacional "La Formación Docente entre el Siglo XIX y el Siglo XXI". Buenos Aires, Argentina.

Maffesoli, M. (2004). El tiempo de las tribus. México: Siglo XXI Editores.

Martínez, B. J. (2001). Arqueología del concepto "compromiso social" en el discurso pedagógico y de formación docente. Revista Electrónica de Investigación Educativa, 3(1). http://redie.uabc.mx/vol3no1/contenidobonafe.html

Pesticide Action Network International (PAN). (2016). Lista de plaguicidas altamente peligrosos de PAN Internacional. https://rap-al.org/wpcontent/uploads/2017/09/HHP-Lista-PAN-2016-actual-traducidaespa $\% \mathrm{C} 3 \% \mathrm{~B} 1 \mathrm{ol} 29 \mathrm{agosto} 17 . \mathrm{pdf}$

Roldan, C. (2012). Lineamientos para la planeación del turismo sostenible en el corregimiento de San Francisco de Asís (Municipio de Acandí, Chocó, Colombia). Revista Gestión y Ambiente, 15(1), 129-142. https:// revistas.unal.edu.co/index.php/gestion/article/view/30827/30935

Santaella, R. E. (2014). Pedagogía crítica, una propuesta educativa para la transformación social. Reidocrea, 3, 147-171. file:///C:/Users/50686/ Downloads/ReiDoCrea-Vol.3-Art.21-Santaella.pdf

Servicio Fitosanitario del Estado. (2016). Sistema de insumos y fiscalización. http://app.sfe.go.cr/SFEInsumos/aspx/Pantallas/PantallaSeleccion.aspx

Téllez, M. (2001). La paradójica comunidad por-venir. En J. Larrosa y Skliar, Habitantes de Babel. Políticas y poéticas de la diferencia. España: Editorial Laertes. 